\title{
Nucleon dissociation and incoherent $J / \psi$ photoproduction on nuclei in ion ultraperipheral collisions at the CERN Large Hadron Collider
}

\author{
V. Guzey, ${ }^{1,2,3}$ M. Strikman, ${ }^{4}$ and M. Zhalov ${ }^{3}$ \\ ${ }^{1}$ Department of Physics, University of Jyväskylä, P.O. Box 35, 40014 University of Jyväskylä, Finland \\ ${ }^{2}$ Helsinki Institute of Physics, P.O. Box 64, 00014 University of Helsinki, Finland \\ ${ }^{3}$ National Research Center “Kurchatov Institute," Petersburg Nuclear Physics Institute (PNPI), Gatchina 188300, Russia \\ ${ }^{4}$ Department of Physics, The Pennsylvania State University, State College, Pennsylvania 16802, USA
}

(Received 3 August 2018; revised manuscript received 5 November 2018; published 10 January 2019)

\begin{abstract}
Using the general notion of cross section fluctuations in hadron-nucleus scattering at high energies, we derive an expression for the cross section of incoherent $J / \psi$ photoproduction on heavy nuclei $d \sigma_{\gamma A \rightarrow J / \psi Y} / d t$, which includes both elastic $d \sigma_{\gamma p \rightarrow J / \psi p} / d t$ and proton-dissociation $d \sigma_{\gamma p \rightarrow J / \psi Y} / d t$ photoproduction on target nucleons. We find that, with good accuracy, $d \sigma_{\gamma A \rightarrow J / \psi Y} / d t$ can be expressed as a product of the sum of the $d \sigma_{\gamma p \rightarrow J / \psi p} / d t$ and $d \sigma_{\gamma p \rightarrow J / \psi Y} / d t$ cross sections, which have been measured at HERA, and the common nuclear shadowing factor, which is calculated using the leading twist nuclear shadowing model. Our prediction for the cross section of incoherent $J / \psi$ photoproduction in $\mathrm{Pb}-\mathrm{Pb}$ ultraperipheral collisions at $\sqrt{s_{N N}}=2.76 \mathrm{TeV}$ and $y=0, d \sigma_{A A \rightarrow J / \psi A Y}(y=0) / d y=0.59-1.24 \mathrm{mb}$ agrees within significant theoretical uncertainties with the data of the ALICE Collaboration.
\end{abstract}

DOI: 10.1103/PhysRevC.99.015201

\section{INTRODUCTION}

It is now widely accepted that ultraperipheral collisions (UPCs) of relativistic ions, which are characterized by scattering at large impact parameters such that the interaction proceeds via emission of quasireal photons, offers unique possibilities to explore photon-photon and photon-hadron (proton, nucleus) interactions at previously unattainable high energies [1]. Focusing on UPC studies of nuclear structure in QCD at the Large Hadron Collider (LHC) for a recent summary, see, e.g., Ref. [2], new constraints on the small- $x$ gluon density in heavy nuclei have been obtained using coherent $J / \psi$ photoproduction in $\mathrm{Pb}-\mathrm{Pb}$ UPCs at $\sqrt{s_{N N}}=2.76 \mathrm{TeV}$ [3-5]. The analysis [6,7] of these data showed that they give first direct and essentially model-independent evidence of large nuclear gluon shadowing $R_{g}=g_{A}\left(x, \mu^{2}\right) /\left[A g_{N}\left(x, \mu^{2}\right)\right] \approx 0.6$ at $x=10^{-3}$ and $\mu^{2}=3 \mathrm{GeV}^{2}$ ( $g_{A}$ and $g_{N}$ are gluon densities in $\mathrm{Pb}$ and the proton, respectively). This is consistent with predictions of the leading twist nuclear shadowing model [8], which have small theoretical uncertainties is this kinematics and agrees with the EPS09 [9], EPPS16 [10], and nCTEQ15 [11] nuclear parton distribution functions, which, however, have significant uncertainties in this kinematic region.

At the same time, predictions of the leading twist nuclear shadowing model significantly underestimate [12] the cross

Published by the American Physical Society under the terms of the Creative Commons Attribution 4.0 International license. Further distribution of this work must maintain attribution to the author(s) and the published article's title, journal citation, and DOI. Funded by $\mathrm{SCOAP}^{3}$. section of incoherent $J / \psi$ photoproduction in $\mathrm{Pb}-\mathrm{Pb}$ UPCs at $\sqrt{s_{N N}}=2.76 \mathrm{TeV}$ and $y=0$ [3]. It was hypothesized in Ref. [12] that the process of $J / \psi$ photoproduction with nucleon dissociation $\gamma N \rightarrow J / \psi Y$, which was not included in that analysis, may contribute to incoherent $J / \psi$ photoproduction on nuclei and improve the agreement between theory and experiment. In this article, we demonstrate that this is indeed the case. Extending the formalism of Ref. [12] to include the $\gamma N \rightarrow J / \psi Y$ contribution to the nuclear incoherent cross section and using the HERA data on the elastic and proton-dissociative $J / \psi$ photoproduction cross sections [13], we show that the predictions of the leading twist nuclear shadowing model for incoherent $J / \psi$ photoproduction in $\mathrm{Pb}-\mathrm{Pb}$ UPCs at $\sqrt{s_{N N}}=2.76 \mathrm{TeV}$ and $y=0$ agree with the available measurement of the ALICE Collaboration.

The rest of the paper is organized as follows. In Sec. II, we introduce nucleon cross section fluctuations and their connection to the elastic and proton-dissociation $J / \psi$ photoproduction cross sections. The derivation of the incoherent cross section of $J / \psi$ photoproduction on nuclear targets using the leading twist nuclear shadowing model and generic representations of hadronic fluctuations of the projectile photon and target nucleons is given in Sec. III. The application of this result to $\mathrm{Pb}-\mathrm{Pb}$ UPCs in the LHC kinematics and comparison to the data of the ALICE Collaboration are presented in Sec. IV. We draw our conclusions in Sec. V.

\section{CROSS SECTION FLUCTUATIONS AND PROTON-DISSOCIATION $J / \psi$ PHOTOPRODUCTION}

At high energies, diffractive dissociation can be understood in the Good-Walker picture in terms of coherent hadronic 
fluctuations, which diagonalize the scattering operator [14]. Applying these ideas to elastic and proton-dissociation $J / \psi$ photoproduction, one obtains for the respective cross sections at the momentum transfer $t=0$,

$$
\begin{aligned}
& \frac{d \sigma_{\gamma p \rightarrow J / \psi p}\left(W_{\gamma p}, t=0\right)}{d t} \\
& =\frac{\varkappa^{2}}{16 \pi}\left(\sum_{i}\left|c_{i}\right|^{2} \sigma_{i}\right)^{2} \equiv \frac{\varkappa^{2}}{16 \pi}\langle\sigma\rangle^{2}, \\
& \frac{d \sigma_{\gamma p \rightarrow J / \psi Y}\left(W_{\gamma p}, t=0\right)}{d t} \\
& =\frac{\varkappa^{2}}{16 \pi}\left[\sum_{i}\left|c_{i}\right|^{2} \sigma_{i}^{2}-\left(\sum_{i}\left|c_{i}\right|^{2} \sigma_{i}\right)^{2}\right] \\
& =\frac{\varkappa^{2}}{16 \pi}\left[\left\langle\sigma^{2}\right\rangle-\langle\sigma\rangle^{2}\right]=\frac{\varkappa^{2}}{16 \pi} \omega_{\sigma}\langle\sigma\rangle^{2},
\end{aligned}
$$

where $\varkappa$ is proportional to the $\gamma-J / \psi$ transition amplitude; $\left|c_{i}\right|^{2}$ and $\sigma_{i}$ are the probability and the corresponding eigenvalue (cross section) for a given fluctuation to contribute to the scattering cross section. Note that index $i$ can also be continuous and multidimensional. The second of Eq. (1) demonstrates that diffractive dissociation is possible only if various fluctuations interact with different $\sigma_{i}$ 's, i.e., the distribution over the fluctuations has a nonvanishing dispersion $\omega_{\sigma}=\left\langle\sigma^{2}\right\rangle /\langle\sigma\rangle^{2}-1[15]$.

Equation (1) is general and admits different interpretations in terms of microscopic models of the nucleon structure. In particular, it was interpreted in terms of fluctuations of the gluon density in the proton in Ref. [16] and fluctuations of the proton shape in Refs. [17-20]. Note also that the latter in the context of the chiral magnetic effects were considered in Ref. [21]; the influence of proton size fluctuations on the number of wounded nucleons was studied in Refs. [22,23].

Extension of Eq. (1) to $t \neq 0$ requires the assumption that the fluctuations do not mix and a specific model for the distribution of fluctuations in the transverse (impact parameter $\vec{b}$ ) plane, see, e.g., Ref. [15]. Indeed, the formalism of cross section fluctuations, which leads to Eq. (1), is applicable only for very small $|t| \ll 1 / R_{T}^{2}$ ( $R_{T}$ is the target size); for larger $t$, the coherence among the eigenstates $i$ implied in Eq. (1) is lost [24], and the dynamics of diffraction dissociation changes. In particular, in the limit of large $|t|>2 \mathrm{GeV}^{2}$, the $\gamma p \rightarrow J / \psi Y$ process may proceed via the perturbative mechanism of the two-gluon exchange [25]. In the following, we assume that the scattering amplitudes corresponding to the elastic and proton-dissociation final states have the following forms in impact parameter space, respectively:

$$
\begin{aligned}
& \Gamma_{N}(\vec{b})=\frac{\langle\sigma\rangle}{4 \pi B_{\mathrm{el}}} e^{-\vec{b}^{2} /\left(2 B_{\mathrm{el}}\right)}, \\
& \Gamma_{Y}(\vec{b})=\frac{\sqrt{\omega_{\sigma}}\langle\sigma\rangle}{8 \pi^{2}} \int d^{2} q_{t}^{\prime} e^{-i \overrightarrow{q_{t}^{\prime}} \vec{b}} f_{\mathrm{pd}}\left(t^{\prime}\right),
\end{aligned}
$$

where $B_{\mathrm{el}}$ is the slope of the elastic cross section; $f_{\mathrm{pd}}(t)$ parametrizes the $t$ dependence of the proton-dissociation cross section [see Eq. (3) below]. Using Eq. (2), Eq. (1) can be generalized to the $t \neq 0$ case as follows:

$$
\begin{aligned}
\frac{d \sigma_{\gamma p \rightarrow J / \psi p}\left(W_{\gamma p}, t\right)}{d t} & =\frac{\varkappa^{2}}{4 \pi}\left|\int d^{2} b e^{i \vec{q}_{t} \vec{b}} \Gamma_{N}(\vec{b})\right|^{2} \\
& =\frac{\varkappa^{2}}{16 \pi}\langle\sigma\rangle^{2} e^{-q_{t}^{2} B_{\mathrm{el}}}, \\
\frac{d \sigma_{\gamma p \rightarrow J / \psi Y}\left(W_{\gamma p}, t\right)}{d t} & =\frac{\varkappa^{2}}{4 \pi}\left|\int d^{2} b e^{i \vec{q}_{t} \vec{b}} \Gamma_{Y}(\vec{b})\right|^{2} \\
& =\frac{\varkappa^{2}}{16 \pi} \omega_{\sigma}\langle\sigma\rangle^{2}\left[f_{\mathrm{pd}}(t)\right]^{2},
\end{aligned}
$$

where $t=-q_{t}^{2}$. Based on our discussion above, Eq. (3) can be viewed as an interpolation between the $t=0$ and the large $|t|$ regimes, whose parameters are determined by available data.

In our analysis, we do not employ a particular dynamical realization for the probabilities $\left|c_{i}\right|^{2}$ in Eqs. (1)-(3) and use instead the $\mathrm{H} 1$ data on elastic and proton-dissociation $J / \psi$ photoproduction [13]. Using these data, we find that $\omega_{\sigma}=$ $0.29 \pm 0.04$ for $m_{p}<M_{Y}<10$ and $40<W_{\gamma p}<110 \mathrm{GeV}$ (these values of $W_{\gamma p}$ overlap with those probed in $\mathrm{Pb}-\mathrm{Pb}$ UPCs at $\sqrt{s_{N N}}=2.76$ at central rapidities). Furthermore, for the measured ratio of the $t$ integrated cross sections, we find $r=\sigma_{\gamma p \rightarrow J / \psi Y}\left(W_{\gamma p}\right) / \sigma_{\gamma p \rightarrow J / \psi p}\left(W_{\gamma p}\right)=0.83 \pm 0.15$ for $\left\langle W_{\gamma p}\right\rangle=93.3 \mathrm{GeV}$; note also that $r$ is a slow function of $W_{\gamma p}$ on the studied interval of $W_{\gamma p}$. The large value of $r$ is a consequence of the fact that the $t$ dependence of $d \sigma_{\gamma p \rightarrow J / \psi_{Y}} / d t$ is much slower than that of $d \sigma_{\gamma p \rightarrow J / \psi p} / d t$. As we show in our numerical analysis in Sec. IV, it is the large cross section of proton-dissociation $J / \psi$ photoproduction on the nucleon (the large value of $r$ ), which increases our theoretical predictions for the cross section of incoherent $J / \psi$ photoproduction on nuclei by almost a factor of two and brings it in agreement with the data of the ALICE Collaboration.

\section{THE CROSS SECTION OF INCOHERENT $\boldsymbol{J} / \psi$ PHOTOPRODUCTION ON NUCLEI}

To include the $\gamma N \rightarrow J / \psi Y$ contribution to the nuclear incoherent cross section, we extend our analysis in Ref. [12] by taking into account the effect of cross section fluctuations discussed in Sec. II. At high energies, the incoherent $\gamma A \rightarrow$ $J / \psi Y$ cross section can be written in the following form (the nuclear final-state $Y$ contains products of nucleus dissociation $A^{\prime}$ caused by the elastic $\gamma N \rightarrow J / \psi N$ and inelastic $\gamma N \rightarrow$ $J / \psi Y$ processes on target nucleons):

$$
\begin{aligned}
& \frac{d \sigma_{\gamma A \rightarrow J / \psi Y}\left(W_{\gamma p}\right)}{d t} \\
& =\frac{\varkappa^{2}}{4 \pi} \sum_{A^{\prime}}\left|\int d^{2} b e^{i \vec{q}_{t} \vec{b}}\left\langle A^{\prime}\left|\Gamma_{A}(b)\right| 0\right\rangle\right|^{2}-\frac{d \sigma_{\gamma A \rightarrow J / \psi A}}{d t} \\
& =\frac{\varkappa^{2}}{4 \pi} \int d^{2} b \int d^{2} b^{\prime} e^{i \vec{q}_{t}\left(\vec{b}-\vec{b}^{\prime}\right)}\left[\left\langle 0\left|\Gamma_{A}^{\dagger}\left(b^{\prime}\right) \Gamma_{A}(b)\right| 0\right\rangle\right. \\
& \left.-\left\langle 0\left|\Gamma_{A}^{\dagger}\left(b^{\prime}\right)\right| 0\right\rangle\left\langle 0\left|\Gamma_{A}(b)\right| 0\right\rangle\right],
\end{aligned}
$$

where $\langle 0|\cdots| 0\rangle$ denotes averaging over the nuclear ground-state wave function; $\Gamma_{A}$ is the nuclear amplitude; $d \sigma_{\gamma A \rightarrow J / \psi_{A}} / d t$ is the coherent nuclear cross section. In the 


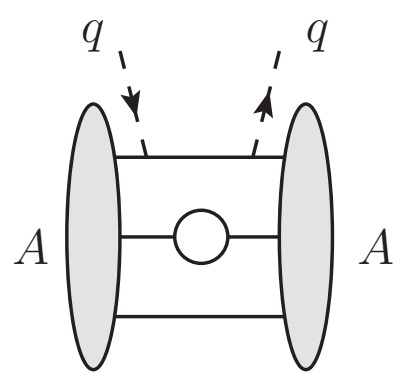

(a)

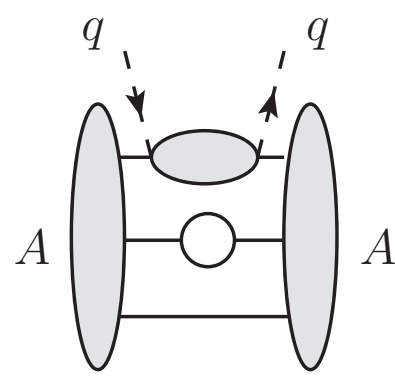

(b)

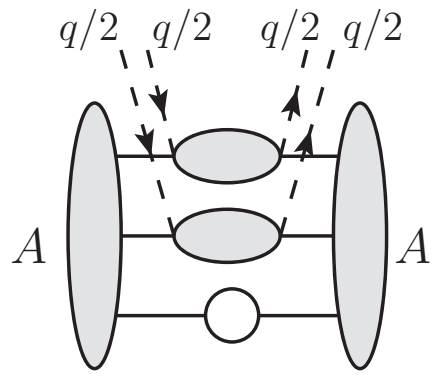

(c)

FIG. 1. Schematic representation of incoherent $J / \psi$ photoproduction on nuclei: (a) contribution of elastic production on nucleons, (b) the proton-dissociation contribution, (c) an example of terms neglected in the last line of Eq. (6). The dashed lines denote the momentum transfer $q$ to the interacting nucleon; the open circles on nucleon lines stand for the interaction with that nucleon leading to attenuation of the resulting cross section; the vertical ovals labeled $A$ denote the nuclear states.

last line of Eq. (4), we used the completeness of nuclear final-states $A^{\prime}$. In the literature [26], this cross section is also called the summed cross section.

The standard representation for $\Gamma_{A}$ in terms of the nucleon scattering amplitudes $\Gamma_{N}$ and $\Gamma_{Y}$ is, see, e.g., Ref. [26],

$$
\Gamma_{A}(\vec{b})=1-\prod_{k=1}^{A}\left[1-\Gamma_{N}\left(\vec{b}-\vec{s}_{k}\right)-\Gamma_{Y}\left(\vec{b}-\vec{s}_{k}\right)\right],
$$

where $\vec{s}_{k}$ denotes the transverse coordinate of the $k$ th nucleon in the nucleus. The nuclear amplitude $\Gamma_{A}(\vec{b})$ is an operator, whose first and second powers [see Eq. (4)] are sandwiched between nuclear ground states. Therefore, only even powers of $\Gamma_{Y}$ contribute to the resulting cross section. Moreover, since powers of $\Gamma_{Y}^{*}\left(\vec{b}^{\prime}-\vec{s}_{k}\right) \Gamma_{Y}\left(\vec{b}-\vec{s}_{k}\right)$ in Eq. (4) involve the same $k$ th nucleon [proton dissociation in $\Gamma_{A}^{\dagger}\left(\overrightarrow{b^{\prime}}\right) \Gamma_{A}(\vec{b})$ takes place on the same nucleon], one does not need to take into account the effects of nucleon ordering and a nonzero longitudinal momentum transfer associated with the final-state $Y$ in Eq. (5). Substituting Eqs. (2) and (5) in Eq. (4) and assuming independent nucleon distributions, one obtains

$$
\begin{aligned}
&\left\langle 0\left|\Gamma_{A}^{\dagger}\left(b^{\prime}\right) \Gamma_{A}(b)\right| 0\right\rangle-\left\langle 0\left|\Gamma_{A}^{\dagger}\left(b^{\prime}\right)\right| 0\right\rangle\left\langle 0\left|\Gamma_{A}(b)\right| 0\right\rangle \\
&= {\left[\left(1-\frac{\langle\sigma\rangle}{2} T_{A}(b)-\frac{\langle\sigma\rangle}{2} T_{A}\left(b^{\prime}\right)+\frac{\langle\sigma\rangle^{2}}{16 \pi B_{\mathrm{el}}} T_{A}(b) e^{-\left(\vec{b}^{\prime}-\vec{b}\right)^{2} /\left(4 B_{\mathrm{el}}\right)}+\frac{\omega_{\sigma}\langle\sigma\rangle^{2}}{16 \pi^{2}} T_{A}(b) \int d^{2} q_{t}^{\prime} e^{i \vec{q}_{t}^{\prime}\left(\vec{b}^{\prime}-\vec{b}\right)}\left[f_{\mathrm{pd}}\left(t^{\prime}\right)\right]^{2}\right)\right]^{A} } \\
&-\left[\left(1-\frac{\langle\sigma\rangle}{2} T_{A}(b)\right)\right]^{A}\left[\left(1-\frac{\langle\sigma\rangle}{2} T_{A}\left(b^{\prime}\right)\right)\right]^{A} \\
& \approx\left(\frac{\langle\sigma\rangle^{2}}{16 \pi B_{\mathrm{el}}} e^{-\left(\overrightarrow{b^{\prime}}-\vec{b}\right)^{2} /\left(4 B_{\mathrm{el}}\right)}+\frac{\omega_{\sigma}\langle\sigma\rangle^{2}}{16 \pi^{2}} \int d^{2} q_{t}^{\prime} e^{i q_{t}^{\prime}\left(\vec{b}^{\prime}-\vec{b}\right)}\left[f_{\mathrm{pd}}\left(t^{\prime}\right)\right]^{2}\right) A T_{A}(b) e^{-\langle\sigma\rangle A T_{A}(b)},
\end{aligned}
$$

where $T_{A}(b)=\int d z \rho_{A}(b, z)$ is the nuclear optical density with $\rho_{A}(b, z)$ being the nuclear distribution. In the derivation of Eq. (6), we used that both $B_{\mathrm{el}}$ and the effective slope of $f_{\mathrm{pd}}(t)$ are much smaller than the slope of the nuclear form factor (the effective nucleus radius) and, hence, the nuclear density can be evaluated at the impact parameter $\vec{b}$. In the last line, we exponentiated the powers of $A$, expanded the result in powers of the elastic and proton-dissociation cross sections, and kept the first leading term. The terms neglected in the last line of Eq. (6) contribute to the $t$-integrated $\sigma_{\gamma A \rightarrow J / \psi Y}\left(W_{\gamma p}\right)$ cross section at the level of a few percent, which is well below the theoretical uncertainty associated with the calculation of the leading contribution to $\sigma_{\gamma A \rightarrow J / \psi Y}\left(W_{\gamma p}\right)$ (see our numerical results in Sec. IV).
In the graphic form, Eq. (6) is schematically shown in Fig. 1 where graph $a$ denotes the contribution proportional to the elastic $J / \psi$ photoproproduction on the proton (nucleon), graph $b$ corresponds to the proton-dissociation contribution (the horizontal ovals denote nucleon dissociation), and graph $c$ is an example of terms proportional to higher powers of the proton dissociation and elastic cross sections, which are neglected in the last line of Eq. (6). Note that these terms have a slower $t$ dependence because as shown in the figure, they involve a fractional momentum transfer. The dashed lines denote the momentum transfer $q$ to the interacting nucleon; the open circles on nucleon lines stand for the interaction with that nucleon, which leads to attenuation of the resulting incoherent cross section (all graphs with $0,1,2, \ldots, A-1$ 
open circles contribute); the vertical ovals labeled $A$ denote the nuclear states.

Substituting Eq. (6) in Eq. (4), we obtain

$$
\begin{aligned}
\frac{d \sigma_{\gamma A \rightarrow J / \psi Y}\left(W_{\gamma p}\right)}{d t} \\
=\frac{\varkappa^{2}}{16 \pi}\left\{\langle\sigma\rangle^{2} e^{-q_{t}^{2} B_{\mathrm{el}}}+\omega_{\sigma}\langle\sigma\rangle^{2}\left[f_{\mathrm{pd}}(t)\right]^{2}\right\} \\
\quad \times \int d^{2} b A T_{A}(b) e^{-\langle\sigma\rangle A T_{A}(b)} \\
=\left(\frac{d \sigma_{\gamma p \rightarrow J / \psi p}\left(W_{\gamma p}, t\right)}{d t}+\frac{d \sigma_{\gamma p \rightarrow J / \psi Y}\left(W_{\gamma p}, t\right)}{d t}\right) \\
\quad \times \int d^{2} b A T_{A}(b) e^{-\langle\sigma\rangle A T_{A}(b)},
\end{aligned}
$$

where in the last line we used Eq. (3). Equation (7) has been derived using standard assumptions of the Gribov-Glauber model of nuclear shadowing and a generic representation of hadronic fluctuations of target nucleons. To include also the effect of hadronic fluctuations in the projective photon, we follow the procedure used in Ref. [12] and express the eikonal factor in Eq. (7) in terms of the cross section $\sigma_{2}$, which is determined by the ratio of the diffractive and usual gluon densities in the proton, and $\sigma_{3} \equiv \sigma_{\text {soft }}$, which is modeled, see details in Ref. [8]. It allows us to rewrite Eq. (7) in the following final form:

$$
\begin{aligned}
& \frac{d \sigma_{\gamma A \rightarrow J / \psi Y}\left(W_{\gamma p}\right)}{d t} \\
& =\left(\frac{d \sigma_{\gamma p \rightarrow J / \psi p}\left(W_{\gamma p}, t\right)}{d t}+\frac{d \sigma_{\gamma p \rightarrow J / \psi Y}\left(W_{\gamma p}, t\right)}{d t}\right) \\
& \quad \times \int d^{2} b A T_{A}(b)\left(1-\frac{\sigma_{2}}{\sigma_{3}}+\frac{\sigma_{2}}{\sigma_{3}} e^{-\left(\sigma_{3} / 2\right) A T_{A}(b)}\right)^{2} .
\end{aligned}
$$

Equation (8) generalizes Eq. (15) of Ref. [12] by including nucleon dissociation and has a clear physical interpretation (see Fig. 1): Photoproduction of $J / \psi$ takes place on all $A$ target nucleons either elastically or with nucleon dissociation; the interaction of photon hadronic fluctuations with remaining nucleons may lead inelastic production; the probability not to have inelastic processes is given by the last term in the brackets in Eq. (8), which describes the effect of nuclear shadowing.

Figure 2 shows separately the $t$ dependence of the two contributions to the $d \sigma_{\gamma A \rightarrow J / \psi Y}\left(W_{\gamma p}\right) / d t$ cross section in Eq. (8): The first term proportional to $d \sigma_{\gamma p \rightarrow J / \psi p} / d t$ is given by the red solid lines, and the second term proportional to $d \sigma_{\gamma p \rightarrow J / \psi Y} / d t$ is given by the blue dot-dashed curves. The corresponding error bands reflect the uncertainty in the calculation of the nuclear shadowing effect, see the discussion in Sec. IV; the value of the invariant photon-nucleon energy $W_{\gamma p}=94 \mathrm{GeV}$ corresponds to the considered case of $\mathrm{Pb}-\mathrm{Pb}$ UPCs at $\sqrt{s_{N N}}=2.76 \mathrm{TeV}$ and $y=0$. This figure clearly demonstrates that, although the elastic contribution dominates at $t \approx 0$, the nucleon-dissociation term wins over for $|t|>$ $0.5 \mathrm{GeV}^{2}$. After integration over $t$, the net contribution of the two terms is numerically close.

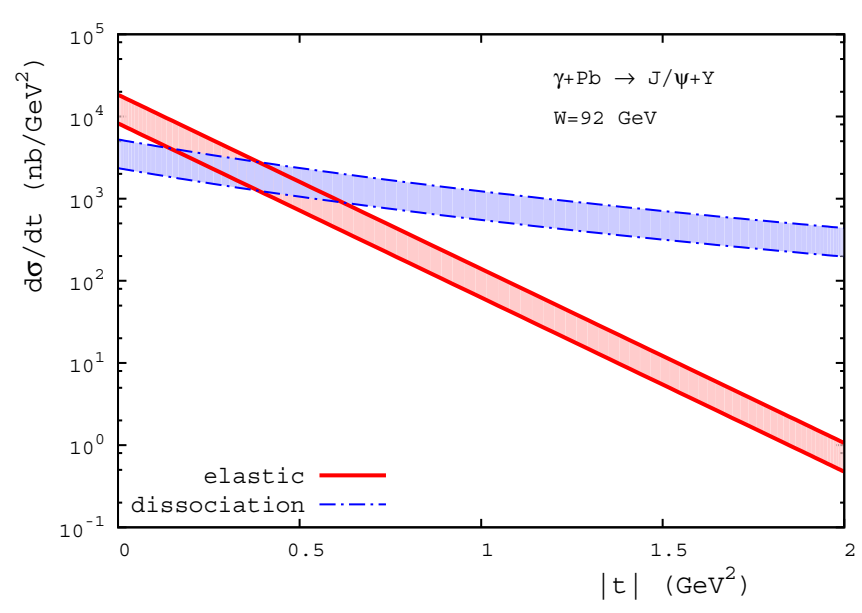

FIG. 2. The elastic and nucleon-dissociation contributions to the $d \sigma_{\gamma A \rightarrow J / \psi Y}\left(W_{\gamma p}\right) / d t$ cross section as a function of $|t|$ corresponding to the first and second terms in Eq. (8), respectively. The shaded error bands quantify the uncertainty in the calculation of nuclear shadowing; $W_{\gamma p}=94 \mathrm{GeV}$ corresponds to Pb-Pb UPCs at $\sqrt{s_{N N}}=$ $2.76 \mathrm{TeV}$ and $y=0$.

Several comments are in order here. First, the Good-Walker picture of hadronic fluctuations (1) is valid strictly only at small $t \approx 0$. In our analysis, to apply completeness of final nuclear states $A^{\prime}$, we implied that $|t|$ is not small. However, there is no contradiction here: The momentum transfer $t$ is indeed small in all vertices except for the one leading to nucleon dissociation (see Fig. 1). For that vertex, we extrapolate to any $t$ using Eq. (3), which allows us to write a simple expression for $d \sigma_{\gamma A \rightarrow J / \psi Y} / d t$ valid for $t \neq 0$. Second, as follows from the second and third lines of Eq. (6), the nuclear incoherent $d \sigma_{\gamma A \rightarrow J / \psi Y} / d t$ cross section in the impulse approximation should vanish in the $t=0$ limit due to a negative (coherent) contribution, which is concentrated at very small $t$; the shadowing correction makes the cross section finite. Since our final expression in Eq. (8) is applied at $|t| \neq 0$, this coherent correction is very small and can be safely neglected. Third, since the slopes of the $t$ dependence of $d \sigma_{\gamma p \rightarrow J / \psi p} / d t$ and $d \sigma_{\gamma p \rightarrow J / \psi Y} / d t$ are very different, the nucleon elastic and dissociation contributions can be separated by studying the $t$ dependence of $d \sigma_{\gamma A \rightarrow J / \psi Y}\left(W_{\gamma p}\right) / d t$.

For the $t$ integrated cross section, one readily obtains from Eq. (8),

$$
\begin{aligned}
\sigma_{\gamma A \rightarrow J / \psi Y}\left(W_{\gamma p}\right) \\
=\left[\sigma_{\gamma p \rightarrow J / \psi p}\left(W_{\gamma p}\right)+\sigma_{\gamma p \rightarrow J / \psi Y}\left(W_{\gamma p}\right)\right] \\
\quad \times \int d^{2} b A T_{A}(b)\left(1-\frac{\sigma_{2}}{\sigma_{3}}+\frac{\sigma_{2}}{\sigma_{3}} e^{-\left(\sigma_{3} / 2\right) A T_{A}(b)}\right)^{2} .
\end{aligned}
$$

One can see from Eq. (9) that the nucleon elastic and dissociation contributions enter with equal nuclear shadowing factors. This can be seen by comparing graphs $a$ and $b$ in Fig. 1 . This result is derived neglecting graph $c$ and other similar graphs, whose net numerical contribution to the $t$-integrated $\sigma_{\gamma A \rightarrow J / \psi Y}\left(W_{\gamma p}\right)$ cross section is a few percent correction. 


\section{INCOHERENT $J / \psi$ PHOTOPRODUCTION IN PB-PB UPCS AND COMPARISON TO DATA OF THE ALICE COLLABORATION}

The cross section of incoherent $J / \psi$ photoproduction in symmetric nucleus-nucleus UPCs reads [1]

$$
\begin{aligned}
\frac{d \sigma_{A A \rightarrow J / \psi A Y}(y)}{d y}= & N_{\gamma / A}(y) \sigma_{\gamma A \rightarrow J / \psi Y}(y) \\
& +N_{\gamma / A}(-y) \sigma_{\gamma A \rightarrow J / \psi Y}(-y)
\end{aligned}
$$

where $N_{\gamma / A}$ is the photon flux; $y$ is the rapidity of the produced vector meson $V ; \sigma_{\gamma A \rightarrow J / \psi Y}(y)$ is the nuclear incoherent cross section integrated over $t$. The presence of two terms with opposite rapidities in Eq. (10) reflects the fact that each colliding ion can serve as a source of photons and as a target. Interference between these two contributions is important only for very small values of $|t|$ and, hence, has been neglected. The photon flux $N_{\gamma / A}(y)$ produced by an ultrarelativistic ion in nucleus-nucleus UPCs is calculated using the standard expressions taking into account the effects of the strong interaction suppression and the nuclear form factor, see, e.g., Ref. [6]. In our analysis, the nuclear density and form factor are calculated using the Hartree-Fock-Skyrme model [27].

Substituting Eq. (9) in Eq. (10), we obtain our prediction for the incoherent cross section $d \sigma_{A A \rightarrow J / \psi_{A Y}}(y=0) / d y$ of $\mathrm{Pb}-\mathrm{Pb}$ UPCs at $\sqrt{s_{N N}}=2.76 \mathrm{TeV}$ at the central rapidity $y=0$. It is shown by the first three lines of Table I, which correspond to the proton elastic contribution, the protondissociation one, and their sum. Each of the values in the first two lines bear $10 \%$ uncertainties due to experimental errors of the respective proton cross sections; the values in the third line come with the combined $15 \%$ experimental uncertainty. Within significant theoretical uncertainties, which we will discuss below, our prediction for $d \sigma_{A A \rightarrow J / \psi A Y}(y=$ $0) / d y$ (third line) agrees with the experimental value of the ALICE Collaboration [3] given in the last line of Table I.

In our numerical analysis, we used the $\mathrm{H} 1$ data [13] on the $\sigma_{\gamma p \rightarrow J / \psi p}$ and $\sigma_{\gamma p \rightarrow J / \psi Y}$ cross sections, see the end of Sec. II. One should note that $r=\sigma_{\gamma p \rightarrow J / \psi Y}\left(W_{\gamma p}\right) / \sigma_{\gamma p \rightarrow J / \psi p}\left(W_{\gamma p}\right)$ depends on the maximal interval of rapidity, which is allowed for the final inelastic state $Y$. Therefore, the cuts used in incoherent $J / \psi$ photoproduction in UPCs should be consistent with those in $\gamma p \rightarrow J / \psi Y$.

The large range of resulting predictions for $d \sigma_{A A \rightarrow J / \psi A Y}(y=0) / d y$ corresponds to the theoretical uncertainty of the leading twist nuclear shadowing model [8], whose largest part is associated with the uncertainty in the effective cross section $\sigma_{3}$. It reflects uncertainties in

TABLE I. Incoherent cross section $d \sigma_{A A \rightarrow J / \psi A Y}(y=0) / d y$ of $\mathrm{Pb}-\mathrm{Pb}$ UPCs at $\sqrt{s_{N N}}=2.76 \mathrm{TeV}$ and $y=0$.

$d \sigma_{A A \rightarrow J / \psi A Y}(y=0) / d y:$ elastic

$d \sigma_{A A \rightarrow J / \psi A Y}(y=0) / d y:$ dissociation

$d \sigma_{A A \rightarrow J / \psi A Y}(y=0) / d y:$ total

Experiment [3]
$0.32-0.68 \mathrm{mb}$

$0.27-0.56 \mathrm{mb}$

$0.59-1.24 \mathrm{mb}$

$0.98_{-0.17}^{+0.19}(\mathrm{sta}+\mathrm{sys}) \mathrm{mb}$ modeling of the interplay between hard and soft components of diffraction in deep inelastic scattering (DIS). At the same time, the parameter $\sigma_{2}$ is constrained much better; a small uncertainty in $\sigma_{2}$ is related to experimental errors of QCD analyses of hard diffraction at HERA, see the details in Ref. [8]. Although these uncertainties lead to approximately $10 \%$ ambiguity in the predicted gluon nuclear shadowing in heavy nuclei at $x \approx 10^{-3}$, they are much larger for hard inelastic diffraction in DIS [8]. As one can see from Table I, incoherent photoproduction of $J / \psi$ on nuclei is also very sensitive to the value of $\sigma_{3}$. Thus, further studies of the discussed process would improve predictions for inclusive diffraction in DIS on nuclei, which is one of key measurements at a future Electron-Ion Collider [28].

To appreciate the magnitude of the leading twist nuclear shadowing suppression, one can cast our results in form of the following ratio:

$$
\begin{aligned}
R & =\frac{\sigma_{\gamma A \rightarrow J / \psi Y}\left(W_{\gamma p}\right)}{A\left[\sigma_{\gamma p \rightarrow J / \psi p}\left(W_{\gamma p}\right)+\sigma_{\gamma p \rightarrow J / \psi Y}\left(W_{\gamma p}\right)\right]} \\
& =\int d^{2} b T_{A}(b)\left(1-\frac{\sigma_{2}}{\sigma_{3}}+\frac{\sigma_{2}}{\sigma_{3}} e^{-\left(\sigma_{3} / 2\right) A T_{A}(b)}\right)^{2} .
\end{aligned}
$$

In the considered kinematics $\left(\sqrt{s_{N N}}=2.76 \mathrm{TeV}\right.$ and $\left.y=0\right)$, we obtain

$$
R=0.13-0.29,
$$

which should be compared to unity in the limit of absence of nuclear shadowing. One can see from Eq. (12) that the effect of nuclear suppression due to the leading twist nuclear shadowing is even stronger than that in the case of coherent $J / \psi$ photoproduction in $\mathrm{Pb}-\mathrm{Pb}$ UPCs $[6,7]$.

It was discussed in the literature that, at large $|t|>2 \mathrm{GeV}^{2}$, the cross section of proton-dissociation $J / \psi$ photoproduction is proportional to the target gluon density $g_{A}(\tilde{x},|t|)$, where $\tilde{x}=-t /\left(-t+M_{Y}^{2}-m_{N}^{2}\right)$ [25]. Hence, in a wide range of $M_{Y}^{2}$ corresponding to $\tilde{x} \geqslant 10^{-2}$ where the effect of nuclear shadowing is weak, one would observe a nearly linear dependence of the cross section on $A$, which is much stronger than that given by Eq. (8) for small $|t|$. At the same time, when $\tilde{x}$ is small, e.g., $\tilde{x} \sim 10^{-3}$, the gluon nuclear shadowing slows down the $A$ dependence of the nuclear cross section. In our approach, $g_{A}\left(x, \mu^{2}\right)$ can be readily evaluated in terms of $\sigma_{2}$ and $\sigma_{3}[12]$,

$$
\begin{aligned}
g_{A}\left(x, \mu^{2}\right)= & A g_{N}\left(x, \mu^{2}\right)\left[1-\frac{\sigma_{2}}{\sigma_{3}}+\frac{2 \sigma_{2}}{A \sigma_{3}^{2}}\right. \\
& \left.\times \int d^{2} b\left(1-e^{-A T_{A}(b)\left(\sigma_{3} / 2\right)}\right)\right],
\end{aligned}
$$

where $g_{N}\left(x, \mu^{2}\right)$ is the gluon density in the nucleon. Despite the leading twist nuclear shadowing effect, the $A$ dependence of $g_{A}\left(x, \mu^{2}\right)$ encoded in Eq. (13) is still much faster than that given by Eq. (8). Indeed, transition to the dominance of the perturbative mechanism should result in a substantial increase of $R(t) \equiv d \sigma_{\gamma A \rightarrow J / \psi Y}\left(W_{\gamma p}\right) / d t /\left[d \sigma_{\gamma p \rightarrow J / \psi p}\left(W_{\gamma p}, t\right) / d t+\right.$ $\left.d \sigma_{\gamma p \rightarrow J / \psi Y}\left(W_{\gamma p}, t\right) / d t\right]$ with an increase in $|t|:$ a factor of $\geqslant 2$ in the discussed kinematics since $g_{A}(\tilde{x},|t|) /\left[A g_{N}(\tilde{x},|t|)\right] \geqslant$ 0.6 due to the leading twist nuclear shadowing. Therefore, 
by studying the $A$ dependence of the $d \sigma_{\gamma A \rightarrow J / \psi Y}\left(W_{\gamma p}\right) / d t$ cross section, one should, in principle, distinguish between the small and large $|t|$ regimes described by Eqs. (8) and (13), respectively.

\section{CONCLUSIONS}

Using the general notion of cross section fluctuations in hadron-nucleus scattering at high energies, we derive an expression for the cross section of incoherent $J / \psi$ photoproduction on heavy nuclei $d \sigma_{\gamma A \rightarrow J / \psi Y} / d t$, which includes both elastic and proton-dissociation processes on target nucleons. The final expression for $d \sigma_{\gamma A \rightarrow J / \psi_{Y}} / d t$ is given in terms of the sum of the $d \sigma_{\gamma p \rightarrow J / \psi p} / d t$ and $d \sigma_{\gamma p \rightarrow J / \psi Y} / d t$ cross sections times the common nuclear shadowing (suppression) factor. Using the HERA data for $\sigma_{\gamma p \rightarrow J / \psi p}$ and $\sigma_{\gamma p \rightarrow J / \psi Y}$ and the results of the leading twist nuclear shadowing model for the suppression factor, we made predictions for the cross section of incoherent $J / \psi$ photoproduction in $\mathrm{Pb}-\mathrm{Pb}$ UPCs at $\sqrt{s_{N N}}=2.76 \mathrm{TeV}$ and $y=0, d \sigma_{A A \rightarrow J / \psi A Y}(y=0) / d y=$ $0.59-1.24 \mathrm{mb}$. Within large theoretical uncertainties of the leading twist nuclear shadowing model for this cross section, our result agrees with the data point of the ALICE Collaboration. The agreement is made possible by the large contribution to the nuclear incoherent cross section of the proton-dissociation process $\gamma p \rightarrow J / \psi Y$. Thus, predictions of the leading twist nuclear shadowing model provide a good description of both coherent and incoherent $J / \psi$ photoproduction in $\mathrm{Pb}-\mathrm{Pb}$ UPCs at the LHC.

\section{ACKNOWLEDGMENTS}

V.G. would like to thank H. Mäntysaari for useful discussions of the results of Refs. [17,18]. The research of M.S. was supported by the U.S. Department of Energy, Office of Science, Office of Nuclear Physics, under Award No. DE-FG0 2-93ER40771.
[1] A. J. Baltz et al., Phys. Rep. 458, 1 (2008).

[2] S. R. Klein, Nucl. Phys. A 967, 249 (2017).

[3] E. Abbas et al. (ALICE Collaboration), Eur. Phys. J. C 73, 2617 (2013).

[4] B. Abelev et al. (ALICE Collaboration), Phys. Lett. B 718, 1273 (2013).

[5] V. Khachatryan et al. (CMS Collaboration), Phys. Lett. B 772, 489 (2017).

[6] V. Guzey, E. Kryshen, M. Strikman, and M. Zhalov, Phys. Lett. B 726, 290 (2013).

[7] V. Guzey and M. Zhalov, J. High Energy Phys. 10 (2013) 207.

[8] L. Frankfurt, V. Guzey, and M. Strikman, Phys. Rep. 512, 255 (2012).

[9] K. J. Eskola, H. Paukkunen, and C. A. Salgado, J. High Energy Phys. 04 (2009) 065.

[10] K. J. Eskola, P. Paakkinen, H. Paukkunen, and C. A. Salgado, Eur. Phys. J. C 77, 163 (2017).

[11] K. Kovařík, A. Kusina, T. Ježo, D. B. Clark, C. Keppel, F. Lyonnet, J. G. Morfín, F. I. Olness, J. F. Owens, I. Schienbein, and J. Y. Yu, Phys. Rev. D 93, 085037 (2016).

[12] V. Guzey, M. Strikman, and M. Zhalov, Eur. Phys. J. C 74, 2942 (2014).

[13] C. Alexa et al. (H1 Collaboration), Eur. Phys. J. C 73, 2466 (2013).
[14] M. L. Good and W. D. Walker, Phys. Rev. 120, 1857 (1960).

[15] H. I. Miettinen and J. Pumplin, Phys. Rev. D 18, 1696 (1978).

[16] L. Frankfurt, M. Strikman, D. Treleani, and C. Weiss, Phys. Rev. Lett. 101, 202003 (2008).

[17] H. Mäntysaari and B. Schenke, Phys. Rev. Lett. 117, 052301 (2016).

[18] H. Mäntysaari and B. Schenke, Phys. Rev. D 94, 034042 (2016).

[19] J. Cepila, J. G. Contreras and J. D. Tapia Takaki, Phys. Lett. B 766, 186 (2017).

[20] M. C. Traini and J.-P. Blaizot, arXiv:1804.06110.

[21] D. Kharzeev, Z. Tu, A. Zhang, and W. Li, Phys. Rev. C 97, 024905 (2018).

[22] M. Alvioli and M. Strikman, Phys. Lett. B 722, 347 (2013).

[23] M. Alvioli, L. Frankfurt, V. Guzey, and M. Strikman, Phys. Rev. C 90, 034914 (2014).

[24] L. L. Frankfurt, G. A. Miller, and M. Strikman, Annu. Rev. Nucl. Part. Sci. 44, 501 (1994).

[25] L. Frankfurt, M. Strikman, and M. Zhalov, Phys. Rev. Lett. 102, 232001 (2009).

[26] T. H. Bauer, R. D. Spital, D. R. Yennie, and F. M. Pipkin, Rev. Mod. Phys. 50, 261 (1978); 51, 407(E) (1979).

[27] M. Beiner, H. Flocard, N. van Giai, and P. Quentin, Nucl. Phys. A238, 29 (1975).

[28] A. Accardi et al., Eur. Phys. J. A 52, 268 (2016). 